\title{
HRM technology for the identification and characterization of INDEL and SNP mutations in genes involved in drought and salt tolerance of durum wheat
}

\author{
Linda Mondini ${ }^{1}$, Miloudi M. Nachit ${ }^{2}$, Enrico Porceddu ${ }^{1}$ \\ and Mario A. Pagnotta ${ }^{1}$. \\ ${ }^{1}$ Department of Agrobiology and Agrochemistry, University of Tuscia, Via S.C. de Lellis, \\ 01100 Viterbo, Italy and ${ }^{2}$ ICARDA, PO Box 5466, Aleppo, Syria
}

\begin{abstract}
WRKY transcription factors are one of the largest families of transcriptional regulators and form an integral part of signalling webs which modulate many plant processes, such as abiotic stress tolerance. In the present paper, an innovative method has been applied to identify novel WRKY-1 alleles involved in the responses to salt and drought stresses in Triticum durum. This technique involves scanning for sequencing variations in CDNA-derived PCR amplicons, using high-resolution melting (HRM) followed by direct Sanger sequencing of only those amplicons which were predicted to carry nucleotide changes. HRM represents a novel advance in detection of single-nucleotide polymorphisms (SNPs) by measuring temperature-induced strand separation of short PCR amplicons. The use of this approach is still limited in the field of plant biology. Here, HRM analysis has been applied to the discovery and genotyping of durum wheat SNPs. Specific primers have been designed, starting at multi-alignment of WRKY-1-conserved portions. The PCR amplicons, containing single SNPs, produce distinctive HRM profiles, and by sequencing the PCR products identified, SNPs have been characterized and validated. The results showed that all the revealed SNPs are located on salt-tolerant varieties, confirming their value in breeding activities.
\end{abstract}

Keywords: durum wheat; high-resolution melting; salt tolerance; single-nucleotide polymorphisms

\section{Introduction}

Environmental stresses, such as salinity, are the cause of great losses in crop yields every year all over the globe (Boyer, 1982). Therefore, salt-responsive or salt-tolerance mechanisms have been intensively studied from biological and genetic perspectives. Signal transduction pathways regulate reactive oxygen species, damage repair and ion homeostasis, while maintaining a low $\mathrm{Na}^{+} / \mathrm{K}^{+}$ ratio is important for plant survival under salt stress (Kader et al., 2006). Molecules that have a function in the adaptation to environmental stresses can be

*Corresponding author. E-mail: pagnotta@unitus.it divided into two groups: the effector molecules and the regulatory molecules (Hasegawa and Bressan, 2000). In particular, WRKY factors are one of the largest families of transcriptional regulators in plants and form integral parts of signalling webs that modulate many plant processes, including the responses to abiotic stresses, such as drought, cold and salt stresses. The WRKY family is among the ten largest families of transcription factors in higher plants and is found throughout the green lineage (Rushton et al., 1996). Recent studies have confirmed that WRKY proteins often act as repressors as well as activators and that the members of the family play a role in both repression and depression of important plant processes (Ishiguro and Nakamura, 1994). Furthermore, it may be that a single WRKY transcription factor is involved in regulating several seemingly disparate 